\title{
Video Article \\ Induction and Analysis of Epithelial to Mesenchymal Transition
}

\author{
Yixin Tang ${ }^{1}$, Greg Herr ${ }^{2}$, Wade Johnson ${ }^{2}$, Ernesto Resnik ${ }^{1}$, Joy Aho ${ }^{2}$ \\ ${ }^{1}$ Antibody Development Department, R\&D Systems, Inc. \\ ${ }^{2}$ Stem Cells and Developmental Biology Department, R\&D Systems, Inc.
}

Correspondence to: Joy Aho at Joy.Aho@RnDSystems.com

URL: https://www.jove.com/video/50478

DOI: doi:10.3791/50478

Keywords: Molecular Biology, Issue 78, Cellular Biology, Biochemistry, Biomedical Engineering, Stem Cell Biology, Cancer Biology, Medicine, Bioengineering, Anatomy, Physiology, biology (general), Pathological Conditions, Signs and Symptoms, Wounds and Injuries, Neoplasms, Diagnosis, Therapeutics, Epithelial to mesenchymal transition, EMT, cancer, metastasis, cancer stem cell, cell, assay, immunohistochemistry

Date Published: 8/27/2013

Citation: Tang, Y., Herr, G., Johnson, W., Resnik, E., Aho, J. Induction and Analysis of Epithelial to Mesenchymal Transition. J. Vis. Exp. (78), e50478, doi:10.3791/50478 (2013).

\section{Abstract}

Epithelial to mesenchymal transition (EMT) is essential for proper morphogenesis during development. Misregulation of this process has been implicated as a key event in fibrosis and the progression of carcinomas to a metastatic state. Understanding the processes that underlie EMT is imperative for the early diagnosis and clinical control of these disease states. Reliable induction of EMT in vitro is a useful tool for drug discovery as well as to identify common gene expression signatures for diagnostic purposes. Here we demonstrate a straightforward method for the induction of EMT in a variety of cell types. Methods for the analysis of cells pre- and post-EMT induction by immunocytochemistry are also included. Additionally, we demonstrate the effectiveness of this method through antibody-based array analysis and migration/invasion assays.

\section{Video Link}

The video component of this article can be found at https://www.jove.com/video/50478/

\section{Introduction}

Epithelial to mesenchymal transition (EMT) is the process by which a polarized epithelial cell undergoes a variety of changes resulting in a highly motile, fibroblast-like mesenchymal cell. This process occurs, in part, through gene expression changes and the degradation of adherens junctions, resulting in an increased ability to migrate and invade. Physiologically, EMT plays important roles during embryonic development and wound healing. Loss of proper control over EMT can lead to fibrosis and metastasis of carcinomas ${ }^{1,2}$

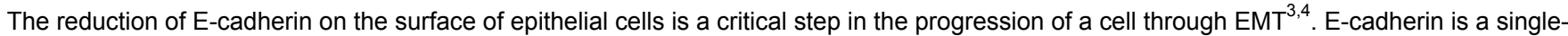
pass transmembrane protein that directly interacts with cadherin proteins on adjacent cells. In addition to its role in cell adhesion, E-cadherin influences cell signaling via interactions between the cytoplasmic tail of E-cadherin and a variety of regulatory proteins, notably $\beta$-catenin. $\beta$-catenin plays a role in the stabilization of adherens junctions. During canonical Wnt signaling, $\beta$-catenin is released from E-cadherin and translocated to the nucleus where it acts as a downstream transcription factor in the Wnt pathway ${ }^{3,4,5}$. In the nucleus, $\beta$-catenin has been shown to increase the transcription of several EMT-related factors including Twist, Slug, Fibronectin, and a variety of matrix metalloproteases ${ }^{3,6}$.

In addition to Wnts, TGF- $\beta$ signaling has been shown to play a significant role in EMT induction both during normal development and in diseased states ${ }^{7,8,9}$. TGF- $\beta$ is involved in the EMT that occurs during palate formation and in the developing heart ${ }^{10}$. It has also been implicated in fibrosis and in cancer progression to metastasis ${ }^{7}$.

The procedure described here provides consistent induction of EMT in a variety of cell types without the need for genetic manipulation. This procedure uses a cocktail of E-cadherin, sFRP-1, and Dkk-1 blocking antibodies and Wnt-5a and TGF- $\beta 1$ recombinant proteins. This cocktail is designed to block E-cadherin-based adhesion while enhancing Wnt and TGF- $\beta$ signaling. The ability for robust EMT induction is critical for understanding the biology of this process and how to manipulate it for therapeutic purposes. Current common markers of EMT, E-cadherin (downregulated in EMT) and Vimentin (upregulated in EMT), can be found co-expressed in cancers and thus do not provide the best diagnostic markers of potential metastatic cells ${ }^{12}$. Importantly, the analysis of a variety of cell types that have undergone EMT is useful to develop common gene expression signatures that can be used for diagnostic purposes in cancer and fibrosis ${ }^{11}$. Additionally, recent studies have demonstrated that EMT may play a role in the formation of cancer stem cells, suggesting that cells that have undergone EMT may be useful for drug screening to identify compounds that can target these cells ${ }^{13}$. 


\section{Induction of EMT}

1. Warm culture media to $37^{\circ} \mathrm{C}$ in a water bath. The culture media used is the same as the media used for standard culture of your cells of interest. For example, the A549 human lung carcinoma cell line is grown in Kaighn's F12 supplemented with 10\% fetal bovine serum and $2 \mathrm{mM}$ glutamine.

2. Harvest cells of interest using a dissociation solution (e.g. TrypLE Express).

3. Suspend cells in pre-warmed culture media in a $15 \mathrm{ml}$ conical tube.

4. Centrifuge the cell suspension at $400 \times \mathrm{g}$ for $5 \mathrm{~min}$.

5. Carefully remove the supernatant by pouring into a waste container.

6. Suspend the cell pellet in pre-warmed culture media.

7. Count viable cells using Trypan Blue. Remove a sample of the cell suspension and dilute in $0.4 \%$ Trypan Blue solution. Place $10 \mu$ of the diluted sample on a hemocytometer and count viable cells (cells that do not turn blue). Use cell counts to determine the cell density in your solution.

8. Plate cells onto tissue culture treated plates or flasks at $0.9-1.0 \times 10^{4}$ cells per $\mathrm{cm}^{2}$. For example, $0.5 \times 10^{6}$ cells plated in a $100 \mathrm{~mm}$ plate in culture media containing 1X EMT Inducing Media Supplement (6 ml media per $100 \mathrm{~mm}$ plate).

9. Culture plated cells in a $37^{\circ} \mathrm{C} / 5 \% \mathrm{CO}_{2}$ incubator. Monitor cell morphology daily.

10. Three days after plating, remove media and replace with fresh pre-warmed culture media containing 1X EMT Inducing Media Supplement. Continue to culture in a $37{ }^{\circ} \mathrm{C} / 5 \% \mathrm{CO}_{2}$ incubator.

11. Five days after plating, cells are ready for analysis. Cell morphology is visualized by inverted light microscopy.

\section{Analysis of Protein Expression by Immunocytochemistry}

1. Prepare sterile $12 \mathrm{~mm}$ coverslips for a 24 -well plate by placing coverslips in a petri dish containing $95 \%$ ethanol. Gently remove coverslips from ethanol using a needle and curved forceps and flame sterilize. Place sterile coverslip into one well of a 24-well plate. Repeat with remaining coverslips.

2. Prepare the cell suspension as described in section $1.1-1.7$.

3. Plate $1.6 \times 10^{4}$ cells / well in $0.5 \mathrm{ml}$ pre-warmed culture media containing 1 X EMT Inducing Media Supplement.

4. Grow and feed cells as described in sections $1.9-1.11$.

5. Five days after plating, remove media and fix cells with $300 \mu \mathrm{l} /$ well of $4 \%$ paraformaldehyde in $1 \mathrm{X}$ phosphate buffered saline (PBS) for 20 min at room temperature.

6. Remove fixative and rinse cells $2 x$ with $1 X$ PBS, $500 \mu l /$ well.

7. Incubate cells in $400 \mu \mathrm{l} /$ well of blocking buffer (1X PBS containing $1 \%$ bovine serum albumin (BSA), $10 \%$ normal donkey serum, and $0.3 \%$ Triton X-100) for $1 \mathrm{hr}$ at room temperature.

8. Incubate in primary antibody at manufacturers recommended concentration in $400 \mu \mathrm{l} /$ well of blocking buffer for $3 \mathrm{hr}$ at room temperature or overnight at $4{ }^{\circ} \mathrm{C}$. When using a primary antibody directly conjugated to a fluorochrome, incubate samples in the dark.

9. Wash cells three times in $500 \mu \mathrm{l} /$ well of $1 \mathrm{X}$ PBS containing $0.1 \%$ BSA for $5 \mathrm{~min}$ each wash. When using a primary antibody directly conjugated to a fluorochrome, proceed directly to step 2.12 .

10. Incubate cells in secondary antibody at manufacturer's recommended concentration in $400 \mu \mathrm{l} /$ well of $1 \mathrm{X}$ PBS containing $1 \%$ BSA for $1 \mathrm{hr}$ at room temperature in the dark.

11. Wash cells three times in $500 \mu \mathrm{l} /$ well of $1 \mathrm{X}$ PBS containing $0.1 \%$ BSA for $5 \mathrm{~min}$ each wash.

12. If desired, counterstain nuclei with DAPI solution.

13. Rinse cells in deionized water and mount coverslips face down on a slide using mounting media.

\section{Representative Results}

The EMT inducing culture conditions described here provide a robust method for the induction of EMT in a variety of cell types. Figures 1 and 2 demonstrate the morphology of and marker expression levels for 4 different human cell lines: T98G glioblastoma cells, HT29 colon adenocarcinoma cells, A549 lung carcinoma cells, and MCF10A mammary epithelial cells. Cells that were treated with the EMT Inducing Media Supplement changed from a classical epithelial morphology (Figures 1A - 1D) to a mesenchymal, spindle-shaped morphology (Figures 1E 1H). EMT-induced cells appeared less densely packed into tight colonies compared to uninduced cells. Uninduced MCF10A samples contained tightly packed clusters surrounded by more loosely packed cells. These tightly packed clusters were E-cadherin positive (Figure 2D) and disappeared upon treatment with the EMT Inducing Media Supplement (Figure 2H).

EMT was also assessed by the downregulation of epithelial markers and upregulation of mesenchymal markers. The downregulation of Ecadherin expression is typically observed following EMT induction in different cell types ${ }^{3,4}$. Figure 2 demonstrates surface expression of Ecadherin in the majority of untreated cells (Figures 2A - 2D; red) in comparison to its absence after EMT induction (Figures 2E - 2H; red). One cell line, T98G, was found to have an extremely low basal level of E-cadherin prior to EMT induction, which precluded its analysis. Upregulation of the mesenchymal marker, Fibronectin, was also visible after induction with the EMT Inducing Media Supplement (Figures 2A - 2D versus Figures 2E - 2H; green).

The expression levels of E-cadherin and Fibronectin seen by immunocytochemistry were further confirmed through western blotting of total cell lysates (Figure 3). The western blot confirmed downregulation of E-cadherin and upregulation of Fibronectin expression seen by immunocytochemistry. The bands were quantitated using densitometry and normalized to GAPDH to determine relative fold change following EMT induction. E-cadherin levels were reduced 6.4 and 3.4 fold in A549 and MCF10A cells, respectively. Fibronectin levels were increased 4.6, 
4.1, and 2.3 fold in T98G, A549, and MCF10A cells, respectively. Although the western blot does not show significant reduction of E-cadherin protein levels in HT29 cells post-EMT induction, the immunocytochemistry results demonstrate a reduction in surface expression of E-cadherin (Figure 2B versus 2F), which is unable to be distinguished in western blotting of total cell lysates.

To further evaluate EMT status, additional known markers for EMT were analyzed pre-and post-EMT induction. Consistent with previous results, E-cadherin was downregulated in all cell lines examined, indicating that EMT was induced. In addition, the mesenchymal markers, Vimentin and Snail, were upregulated in A549, T98G, and MCF10A cells following treatment with EMT Inducing Media Supplement (Figure 4). The HT29 cell line did not show expression of these mesenchymal markers either with or without EMT induction (data not shown), which may indicate that these cells utilize alternate pathways for EMT induction.

Although TGF- $\beta$ is sufficient to induce EMT in certain cell contexts ${ }^{7,8,9}$, other cell types do not respond solely to the addition of TGF- $\beta^{14}$. MCF7 human breast cancer cells and PANC-1 human pancreatic carcinoma cells are both reported not to respond to TGF- $\beta$ signaling alone ${ }^{14}$. To determine whether both lines could undergo EMT in vitro, cells were cultured in the presence of the EMT Inducing Media Supplement. Cells were stimulated with recombinant TGF- $\beta 1$ alone, at the concentration within the EMT Inducing Media Supplement. These cells retained their epithelial morphology, consisting of tightly packed colonies of cells (data not shown) and surface E-cadherin levels were similar to those seen in control cells (Figures 5C and 5D versus Figures 5A and 5B). In contrast, cells stimulated with the EMT Inducing Media Supplement showed a drastic decrease in surface E-cadherin levels (Figures $\mathbf{5 E}$ and $\mathbf{5 F}$ ). These cells also obtained a more mesenchymal morphology, as cells became less compact and more spindle-like (data not shown). These results demonstrate that the EMT inducing method described here is able to promote EMT morphology and marker expression changes in cells typically resistant to TGF- $\beta$-induced EMT.

In addition to changes in marker expression, another hallmark of mesenchymal cells is their ability to migrate and invade. Migration and invasion of cells cultured with or without the EMT Inducing Media Supplement were analyzed using the 96 well BME Cell Invasion Assay according to the manufacturer's instructions. Briefly, cells were seeded onto 96-well plates (50,000 cells/well) containing filter inserts with $8.0 \mu \mathrm{m}$ pores. After 48 $\mathrm{hr}$, migration was assessed by calcein AM staining of cells that had migrated through the filter. Each sample was tested in triplicate twice with similar results. Representative results from one experiment are shown in Figure 6A. Statistically significant increases $(P-v a l u e<0.003)$ in cell migration were seen with both $\mathrm{A} 549$ and PANC-1 cells following EMT induction. To assess the ability of these cells to invade (i.e. migrate through extracellular matrix), the same assay was performed with a basement membrane extract-coated filter. EMT-induced cells showed a statistically significant $(P$-value $<0.001)$ increase in invasion capabilities compared to untreated cells as seen in Figure $6 \mathbf{B}$.

The robust induction of EMT is useful for the analysis of gene expression changes and signaling taking place in these cells. Antibody-based array analysis using lysates from both treated and untreated cells is one method to analyze the expression levels of a variety of molecules in a single sample. As an example, we analyzed the levels of phosphorylated MAPK family members in lysates from un-induced and EMT-induced MCF7 and A549 cells using the Proteome Profiler Human Phospho-MAPK Array Kit. The array was run twice from independent EMT-induction treatments with similar results. Figure 7 shows representative data from one array. Both cell types exhibited increased phosphorylation of CREB (S133), ERK1 (T202/Y204), and ERK2 (T185/Y187) in EMT-induced cells compared to controls. Differences in signaling events were observed between the two cell types as well. A549 cells showed an increase in GSK-3 $\beta$ (S9) phosphorylation, whereas MCF7 cells showed increased p70S6K (T421/S424) phosphorylation.
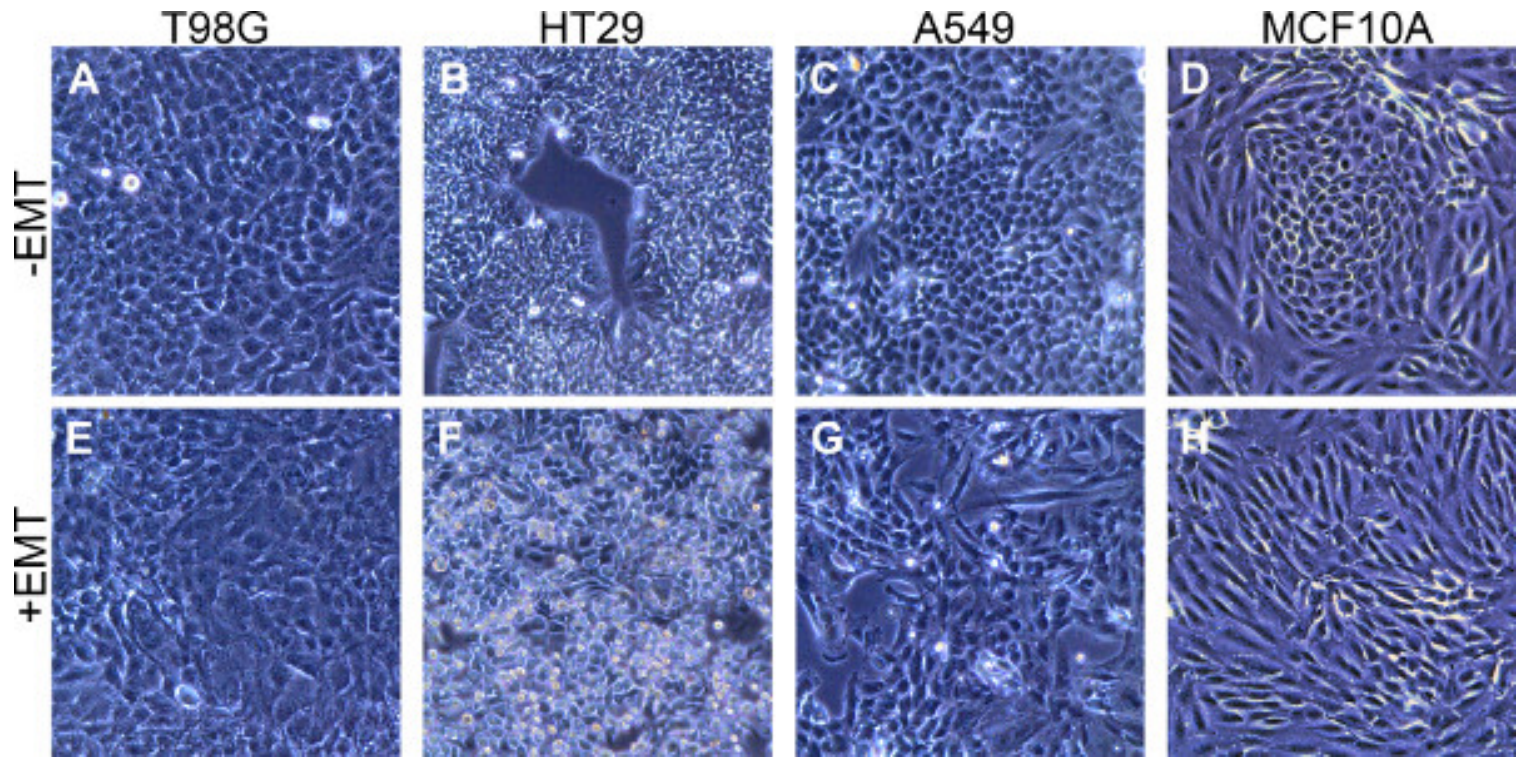

Figure 1. Mesenchymal morphology induction after stimulation with the EMT Inducing Media Supplement. (A - D) Epithelial morphology in the four cell types indicated following culture for 5 days in standard culture media without the EMT Inducing Media Supplement. (E - H) Mesenchymal morphology in the four cell types indicated cultured with the EMT Inducing Media Supplement for 5 days. 

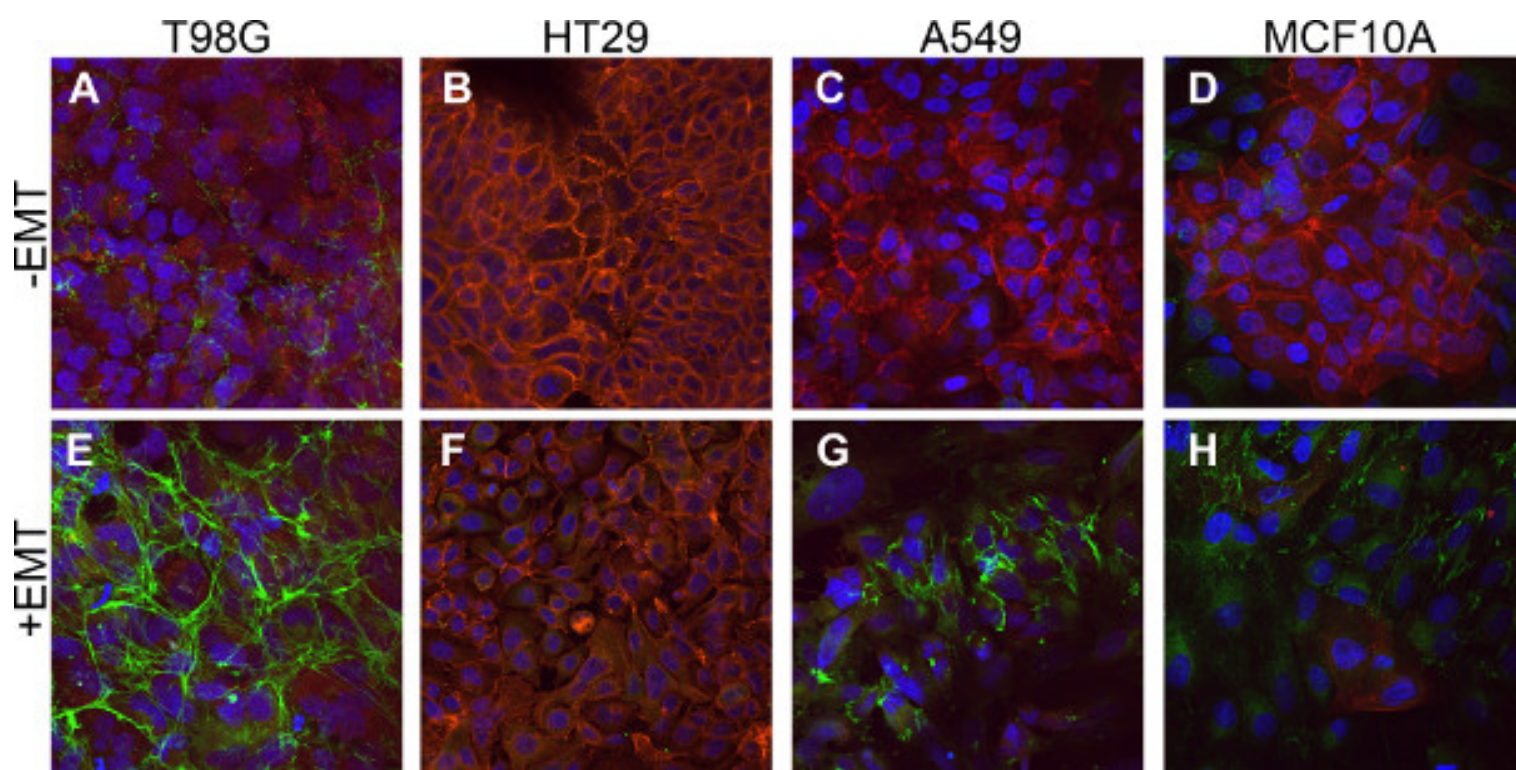

Figure 2. Downregulation of epithelial marker expression and upregulation of mesenchymal marker expression in EMT-induced cells. Cells were stained by immunofluorescence to detect the expression of the epithelial marker, E-cadherin (red), and the mesenchymal marker, Fibronectin (green). (A - D) Control cells cultured without the EMT Inducing Media Supplement for 5 days. (E - H) Cells cultured with the EMT Inducing Media Supplement for 5 days. All cells are counterstained with DAPI (blue).

\section{$\frac{\mathrm{T} 98 \mathrm{G}}{-\mathrm{EMT}} \frac{\mathrm{HT} 29}{-\mathrm{EMT}} \frac{\mathrm{A} 549}{-\mathrm{EMT}} \frac{\mathrm{MCF} 10 \mathrm{~A}}{-\mathrm{EMT}}$}

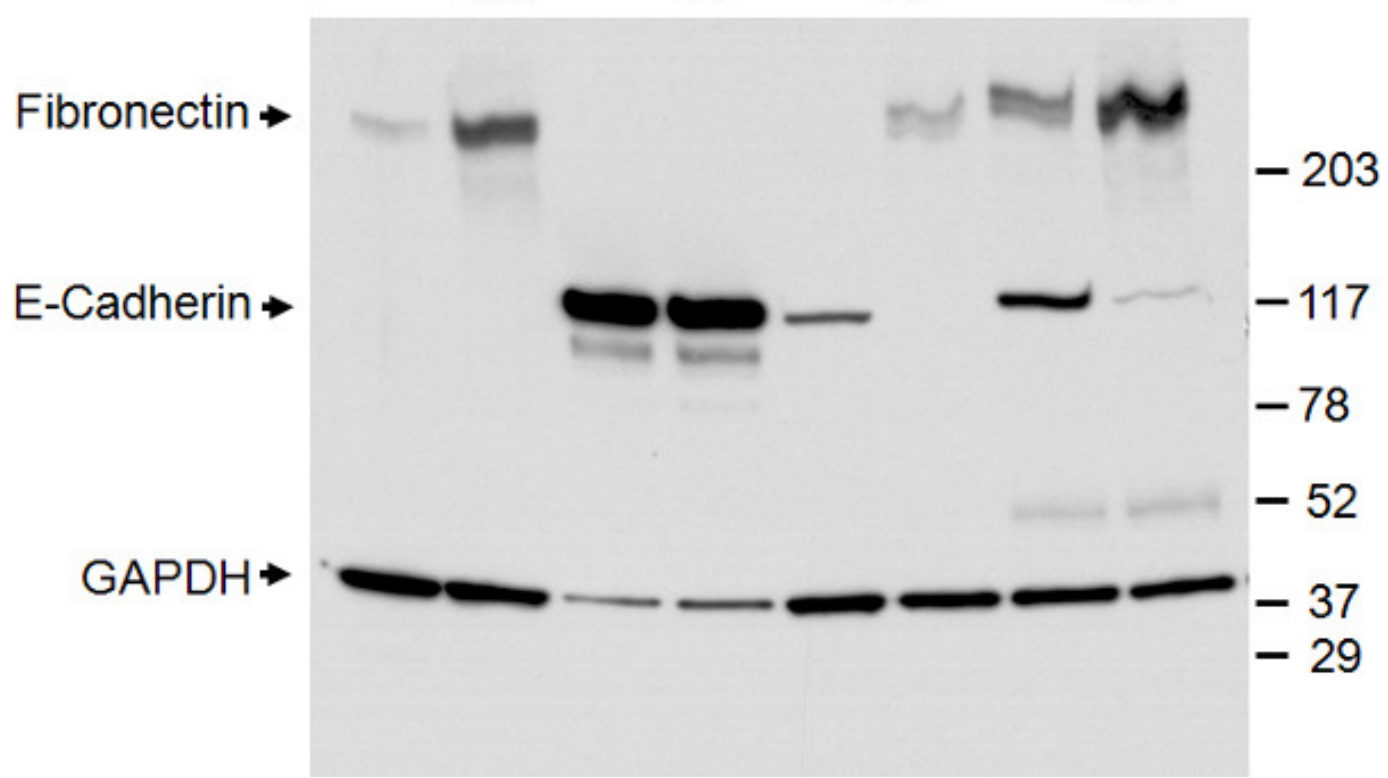

Figure 3. Confirmation of epithelial and mesenchymal marker expression with western blot analysis. Western blot of total cell lysates of the four cell types indicated, treated with (EMT) or without (-) the EMT Inducing Media Supplement for 5 days. Bands for E-cadherin, Fibronectin, and the GAPDH loading control are as indicated on the left. The size marker is as indicated on the right. 

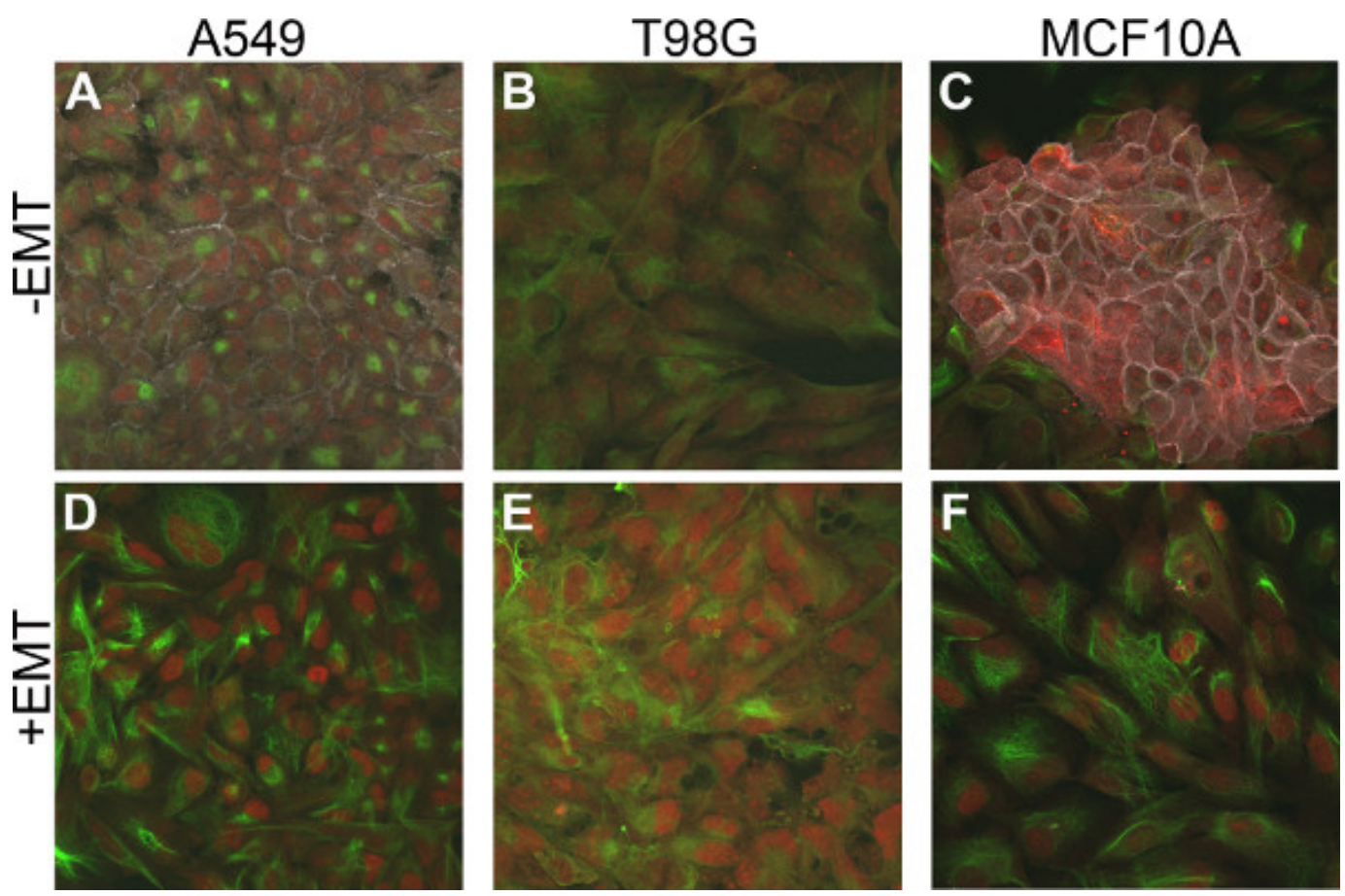

Figure 4. Upregulation of the mesenchymal markers, Vimentin and Snail in EMT-induced cells. Cells were stained by multicolor immunofluorescence to detect expression of the epithelial marker, E-cadherin (gray), and the mesenchymal markers, Vimentin (green) and Snail (red). (A - C) Control cells cultured without the EMT Inducing Media supplement for 5 days. (D - F) Cells cultured with the EMT Inducing Media Supplement for 5 days. 


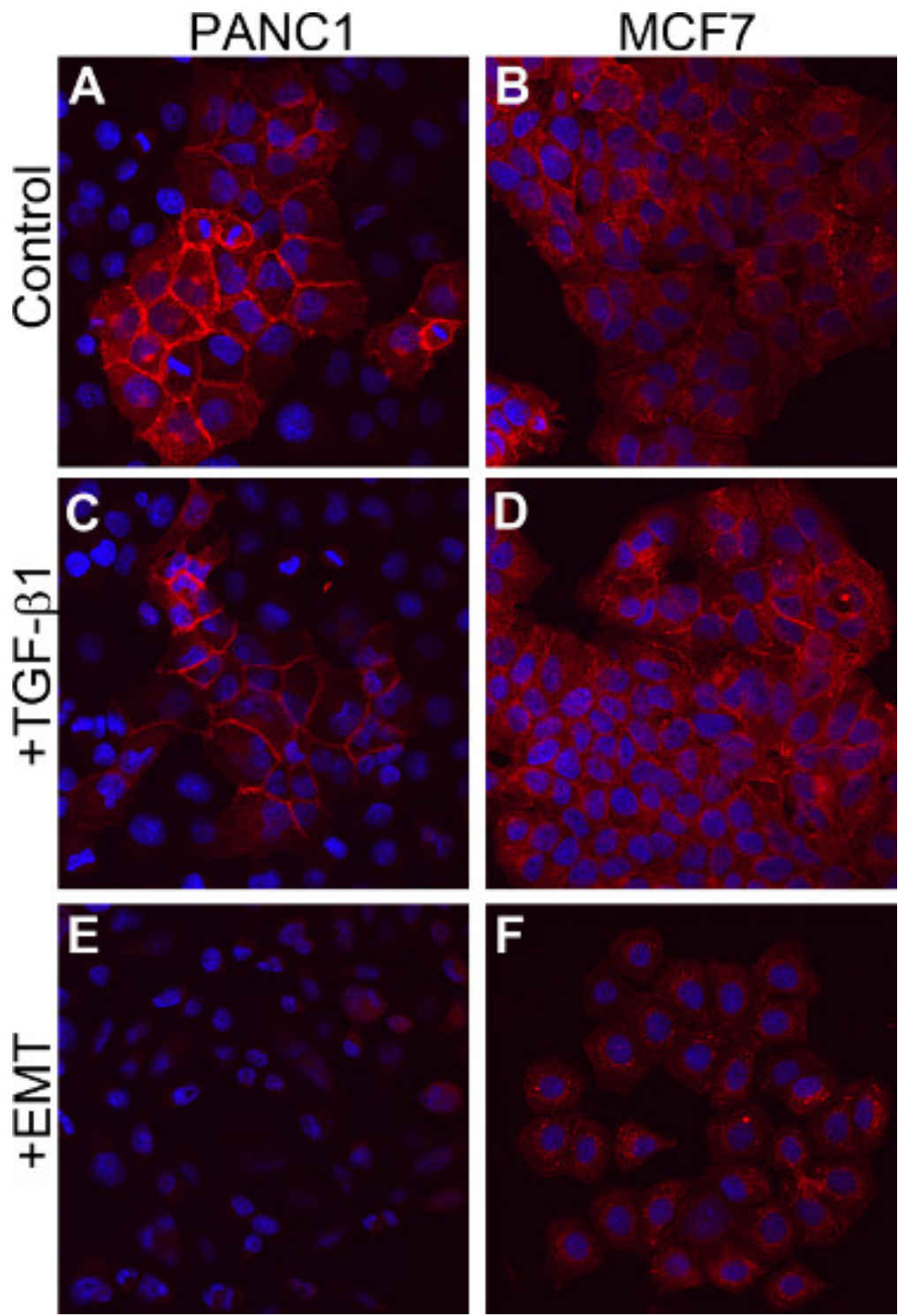

Figure 5. Induction of EMT in TGF- $\beta 1$ non-responsive cells. (A and B) Cells cultured with media alone for 5 days. (C and D) Cells cultured in media containing $10 \mathrm{ng} / \mathrm{ml}$ of TGF- $\beta 1$ for 5 days. (E and F) Cells cultured in EMT Inducing Media Supplement for 5 days. All cells are stained for E-cadherin expression (red) and counterstained with DAPI (blue). 


\section{A Increased migration of EMT-induced cells}

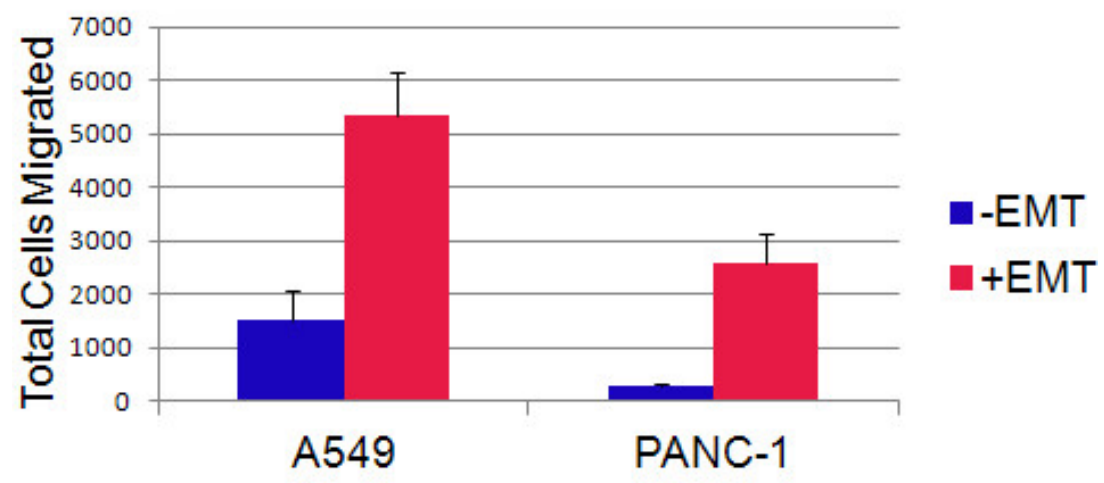

\section{B Increased invasion of EMT-induced cells}

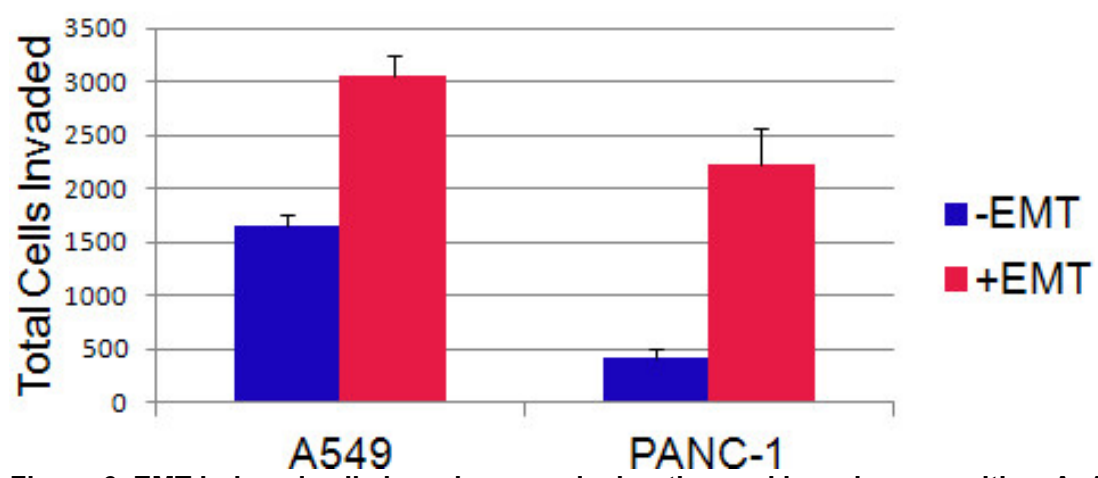

Figure 6. EMT-induced cells have increased migration and invasion capacities. A. Average number of cells that migrated through an 8 $\mu \mathrm{m}$ pore filter after incubation for $48 \mathrm{hr}$. B. Average number of cells that were able to invade through an $8 \mu \mathrm{m}$ pore filter coated with basement membrane extract after incubation for $48 \mathrm{hr}$. Error bars indicate the standard deviation over 3 wells. 
A

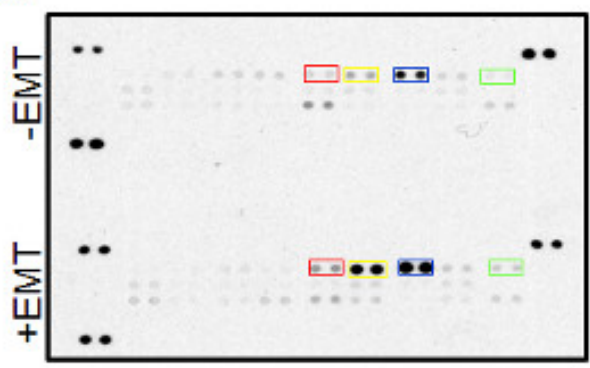

C

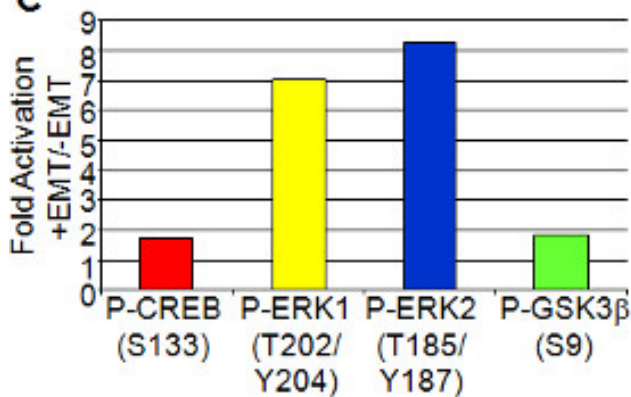

B

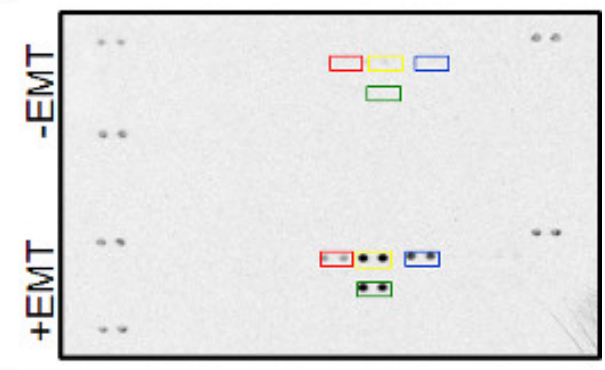

D

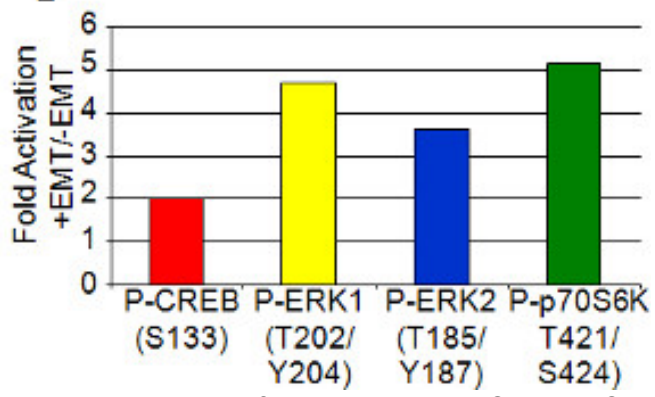

Figure 7. Antibody-based array expression analysis of EMT induction. Lysates from A549 (A and C) and MCF7 (B and D) cells cultured with or without EMT Inducing Media Supplement were used for array analysis using the Proteome Profiler Human Phospho-MAPK Array Kit. Spots highlighted in A and B were then quantitated using densitometry. Comparisons of un-induced to EMT-induced lysates are shown in the corresponding bar graphs below (C and D). Click here to view larger figure.

\section{Discussion}

Previous methods for EMT induction have generally used TGF- $\beta$ stimulation or genetic modification. Although TGF- $\beta$ alone has been shown to induce EMT in some cell types, it has now been demonstrated that TGF- $\beta$ is necessary for EMT, but it is not sufficient in all cell types ${ }^{6,14}$. Using genetic modification for EMT induction is time consuming and labor intensive. The method described here uses a combination of factors including, anti-human E-cadherin, anti-human sFRP-1, anti-human Dkk-1, recombinant human Wnt-5a, and recombinant human TGF- $\beta 1$ to reliably induce EMT. This combination of factors is designed to block E-cadherin-based adhesion, a critical step for EMT induction ${ }^{4}$, and enhance Wnt signaling by adding the Wnt-5a protein as well as using blocking antibodies to the Wnt inhibitors, sFRP-1 and Dkk-1. Wnt and TGF- $\beta$ signaling have been shown to act in an autocrine loop to induce and maintain the mesenchymal cell state ${ }^{6}$. This induction method avoids genetic manipulation and is more effective than using TGF- $\beta$ alone. Importantly, we are able to observe induction of EMT in cell types, including MCF7 and PANC-1 cells, that have been previously shown not to respond solely to TGF- $\beta$ stimulation (Figure 5) ${ }^{14}$.

Cells treated with the EMT Inducing Media Supplement show less compact and increased spindle-shaped morphology (Figure 1). Additionally, there is a decrease in surface E-cadherin expression concurrent with an increase in Fibronectin expression, which are hallmarks of EMT (Figures 2 and 3). Additional mesenchymal markers such as Vimentin and Snail are also upregulated in the EMT induced cells as compared to uninduced controls (Figure 4). Increased migration and invasion abilities are the main functional characteristics of mesenchymal cells. In in vitro migration and invasion assays, cells treated with the EMT Inducing Media Supplement demonstrated a significantly increased capacity to migrate and invade (Figure 6).

This simple method for EMT induction is useful for the study of EMT signaling as demonstrated using the antibody array expression data shown in Figure 7. Different cell types can be compared pre- and post-EMT induction to obtain common expression signatures associated with EMT, such as the increase in phosphorylated CREB and ERK1/2 seen in both MCF7 and A549 EMT-induced cells. S133 phosphorylation of CREB stimulates DNA binding and has been shown to act downstream of TGF- $\beta 1$-induced EMT ${ }^{15}$. ERK1/2 phosphorylation has also been shown to act downstream of TGF- $\beta 1$-induced $\mathrm{EMT}^{16}$. Differences in signaling pathways among cells types can also be elucidated as seen with the increase in GSK-3 $\beta$ phosphorylation in A549 cells versus p70S6K phosphorylation in MCF7. GSK-3 $\beta$ phosphorylation at serine 9 decreases it function, and GSK-3 $\beta$ has been shown to inhibit the pro-EMT transcription factor, Snail ${ }^{17}$. In contrast, p70S6K induces Snail activity and its phosphorylation is associated with activation of this protein ${ }^{18}$.

Overall, straightforward and reliable induction of EMT, particularly in cells previously shown not to respond solely to TGF- $\beta$, is useful for understanding the biology of EMT, identifying common expression signatures for use as prognostic markers, and developing and evaluating novel therapeutics for cancer and fibrotic disease.

\section{Disclosures}

R \& D Systems, Inc. is a for-profit, public company.

Production and Free Access to this article is sponsored by R \& D Systems, Inc. 


\section{Acknowledgements}

The authors would like to acknowledge Julia Hatler and Martin Ramsden (R \& D Systems) for helpful discussion and manuscript review.

\section{References}

1. Kalluri, R. \& Weinberg, R.A. The basics of epithelial-mesenchymal transition. Journal of Clinical Investigation. 119, 1420-1428 (2009).

2. Thiery, J.P. Epithelial-mesenchymal transitions in tumour progression. Nature Reviews Cancer. 2, $442-454$ (2002).

3. Heuberger, J. \& Birchmeier, W. Interplay of cadherin-mediated cell adhesion and canonical Wnt signaling. Cold Spring Harbor Perspectives in Biology. 2, 1-23 (2010).

4. Onder, T.T., et al. Loss of E-cadherin promotes metastasis via multiple downstream transcriptional pathways. Cancer Research. 68, 3645-3654 (2008).

5. Tian, X., et al. E-cadherin/B-catenin complex and the epithelial barrier. Journal of Biomedicine and Biotechnology. 2011, 1-6 (2011)

6. Scheel, C., et al. Paracrine and autocrine signals induce and maintain mesenchymal and stem cell states in the breast. Cell. 145, 926-940 (2011).

7. Xu, J., Lamouille, S., \& Derynck, R. TGF- $\beta$-induced epithelial to mesenchymal transition. Cell Research. 19, 156-172 (2009).

8. Kasai, H., et al. TGF- $\beta 1$ induces human alveolar epithelial to mesenchymal cell transition (EMT). Respiratory Research. 6, 56-70 (2005).

9. Miettinen, P.J., et al. TGF- $\beta$ induced transdifferentiation of mammary epithelial cells to mesenchymal cells: involvement of type I receptors. Journal of Cell Biology. 127, 2021-2036 (1994).

10. Pelton, R.W., et al. Differential expression of genes encoding TGFs $\beta 1, \beta 2$, and $\beta 3$ during murine palate formation. Dev. Biol. 141, 456-460 (1990).

11. Taube, J.H., et al. Core epithelial-to-mesenchymal transition interactome gene-expression signature is associated with claudin-low and metaplastic breast cancer subtypes. PNAS. 107, 15449-15454 (2010).

12. Roussos, E.T., et al. AACR special conference on epithelial-mesenchymal transition and cancer progression and treatment. Cancer Research. 70, 7360-7364 (2010).

13. Polyak, K. \& Weinberg, R.A. Transitions between epithelial and mesenchymal states: acquisition of malignant and stem cell traits. Nature Reviews Cancer. 9, 265-273 (2009).

14. Brown, K.A., et al. Induction by transforming growth factor- $\beta 1$ of epithelial to mesenchymal transition is a rare event in vitro. Breast Cancer Research. 6, R215-R231 (2004).

15. De Falco, V., et al. CD44 proteolysis increases CREB phosphorylation and sustains proliferation of thyroid cancer cells. Cancer Research. 72 , 1449-1458 (2012).

16. Xie, L., et al. Activation of the Erk pathway is required for TGF- $\beta 1$-induced EMT in vitro. Neoplasia. 6, 603-610 (2004).

17. Bachelder, R.E., et al. Glycogen synthase kinase-3 is an endogenous inhibitor of Snail transcription: implication for the epithelialmesenchymal transition. Journal of Cell Biology. 168, 29-33 (2005).

18. Pon, Y.L., et al. p70 S6 kinase promotes epithelial to mesenchymal transition through Snail induction in ovarian cancer cells. Cancer Research. 68, 6524-6532 (2008).

19. Wegner, G.J., et al. Profiling changes in receptor tyrosine kinase phosphorylation using antibody arrays. JoVE. (2012). 